\title{
Designing of a 2x2 E-shaped Microstrip Patch Grid Antenna
}

\author{
JUHI K. BARUAH, KANDARPA KUMAR SARMA, SivarANJAN GOSWAMI \\ Department of Electronics and Communication Engineering Gauhati University Guwahati, INDIA
}

\begin{abstract}
In this work, a $2 \times 2$ grid of E-shaped patch antennas is proposed. The design of the grid is achieved through the design of a single element, the design of a $1 \times 2$ array and finally the design of the $2 \times 2$ grid on an FR 4 epoxy substrate of thickness $1.5 \mathrm{~mm}$. A corporate feed network of microstrip lines is used to excite the array. The performance of each stage is studied in terms of the return loss parameter, the far field gain, and the beam-widths are observed in each case from simulation results. The resonant frequency in each case is $3.8 \mathrm{GHz}$. It is observed that as the number of elements is increased, the beam-width reduces. In other words, the directivity is increased. Further, it is also observed that the gain and bandwidth is the minimum for the single patch, followed by that of the $1 \times 2$ array and the maximum for the $2 \times 2$ grid. Thus, the construction of the grid leads to increase in gain, bandwidth and directivity of the antenna.
\end{abstract}

Keywords - Microstrip patch antenna, 3.8GHz, stub matching, $2 \times 2$ grid pattern, Antenna gain

Received: February 2, 2021. Revised: July 31, 2021. Accepted: August 5, 2021. Published: August 10, 2021.

\section{Introduction}

Microstrip patch antennas have received significant attention worldwide in the past few decades because of its small size, low profile, light weight, low production cost and other aspects of the advantage[1][2]. Today it is widely being used in mobile communications, satellite communications, radar, and other various fields [3]. The performance of an antenna can be enhanced in terms of different parameters by constructing an array of microstrip antennas. Till now various array forms of microstrip patch antennas have been designed [4]-[7] for various applications such as GSM, radar etc. An array can be designed as a one dimensional array consisting of antenna elements along a line or it can be designed in a grid pattern by placing equal numbers of patch in row and coloumn wise as in [3][4]. In addition to the basic advantages of the microstrip patch antenna, the grid pattern antenna has the advantages of high gain, high bandwidth, narrow focused beam, low sidelobe and radiation characteristics [9].

In this paper a $2 \times 2$ microstrip patch grid antenna is designed at $3.8 \mathrm{GHz}$ frequency. The antenna is having an Eshaped patch, as it has a larger bandwidth as compared to a rectangular patch [10]. The antenna is designed with a single patch first and then it is transformed to a $1 \times 2$ array. Finally a $2 \times 2$ grid of microstrip patch antennas is derived out by combining two $1 \times 2$ arrays. Initially, a quarter wavelength transformer is used for impedance matching in the $1 \times 2$ array. A stub matching technique is used in case of the $2 \times 2$ grid to ensure that return loss is below $-15 \mathrm{~dB}$.

The design procedure of all the three configurations: the single patch, the $1 \times 2$ array, and the $2 \times 2$ grid is discussed in section II. Se ction III includes the simulation and measurement results and discussions of the work. Finally, the conclusions are made in section IV.

\section{Design Procedure}

The design procedure involves the design of the single patch antenna element followed by the design of a $1 \times 2$ array and finally a $2 \times 2$ grid of microstrip antennas. FR4 epoxy substrate with a dielectric constant, $\varepsilon_{\mathrm{r}}$ of 4.4 and a thickness of $1.5 \mathrm{~mm}$ is used for the designs. The details of these design steps are discussed as follows.

\subsection{Design of a Single Patch E-shaped Antenna}

The topology of the E-shaped patch antenna element and its various geometrical parameters are shown in Figure 1. The dimensions are experimentally tuned with Ansys HFSS to obtain resonance at $3.8 \mathrm{GHz}$ with a return loss of $-16 \mathrm{~dB}$. The final dimensions of the patch are shown in Table 1.

w

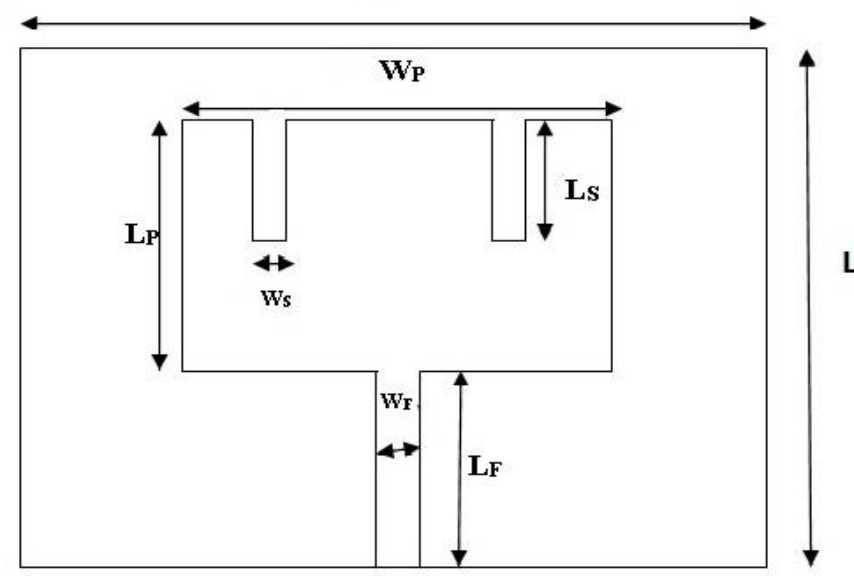

Fig.1 Micro strip single patch E- shaped antenna 
WSEAS TRANSACTIONS On COMMUNICATIONS DOI: 10.37394/23204.2021.20.16

TABLE I. DIMENSIONS OF SINGLE PATCH ANTENNA

\begin{tabular}{|l|l|}
\hline Specifications & Dimensions (in mm) \\
\hline Length of the substrate, L & 60 \\
\hline Width of the substrate, W & 60 \\
\hline Length of patch, LP & 18.4 \\
\hline Width of patch, WP & 25 \\
\hline Feed width, WF & 2.868 \\
\hline Slot length, Ls & 0.8 \\
\hline Slot width, Ws & 0.5 \\
\hline
\end{tabular}

\section{B. $1 \times 2$ Patch Antenna design}

The $1 \times 2$ array is designed using the E-shaped patch antenna as elements. The dimensions of the patch are kept the same so that the array also resonates at $3.8 \mathrm{GHz}$. In this design a corporate feeding network is used. The patch elements are fed in parallel using microstrip transmission lines. These lines are divided into two branches in order to match the impedance of patch elements. The power divider accomplished by using quarter wavelength transformer [11] with $70.71 \mathrm{ohm}$ to match $100 \mathrm{ohm}$ to $50 \mathrm{ohm}$ as in [6]. The patch spacing kept is $\lambda / 4$ that is $19.723 \mathrm{~mm}$. Fig. 2 shows the design view of the specified.

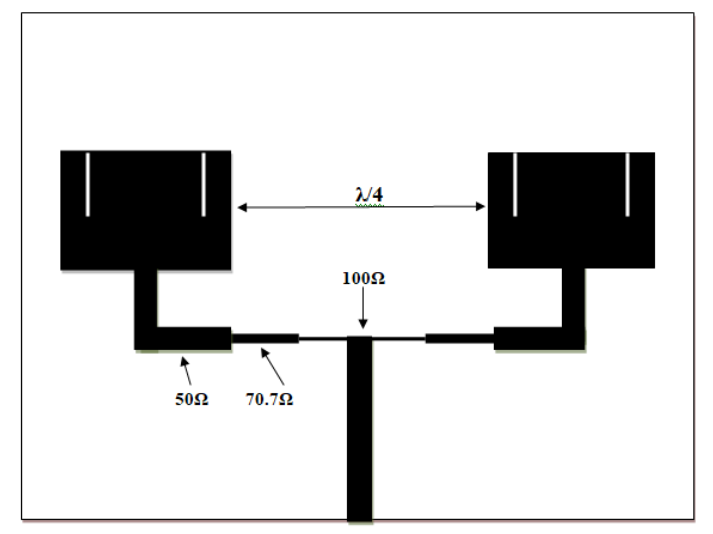

Fig.2. Layout of 1x 2 patch antenna

\section{C. $2 \times 2$ Grid micro strip patch design}

In the design of $2 \times 2$ micro strip patch grid antenna, four patch elements are used in a grid shape as shown in Figure 3. The feed line splits into two branhes as in case of the $1 \times 2$ array. Each branch further splits into two sub-branches each of which excites one E-shaped patch antenna as in [7]. Quarter wavelength transformer technique is used in each split to ensure proper impedance matching. Each element has the same dimensions as discussed in Section II (a) above.

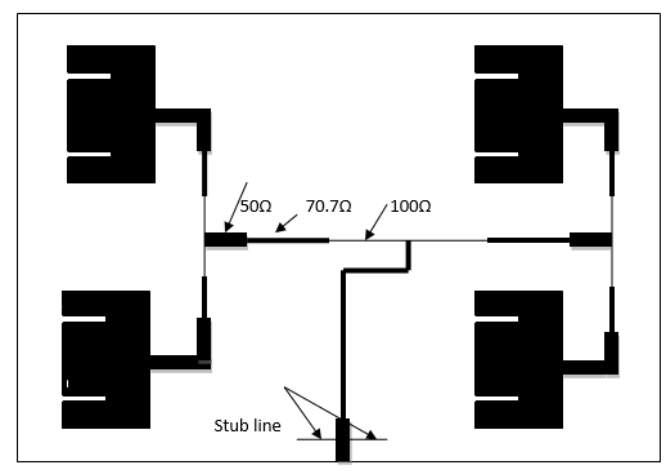

Fig.3.Layout of $2 \times 2$ Micro strip patch Grid antenna

Although quarter wavelength transformers are used at each split of the corporate feeding network, experimentally it was observed that the impedance was not perfectly matched and the return loss was above $-10 \mathrm{~dB}$. To further improve the impedance matching, stub lines are used just near the feed position of the corporate network as shown in Figure 3. Each of the two stub lines has a width of $0.5 \mathrm{~mm}$ and length of 5 mm. A similar approach for impedance matching was reported in [5].

\section{Results and Discussion}

The single patch antenna, the $1 \times 2$ array and the $2 \times 2$ grid are designed and simulated to analyze the results. The final $2 \times 2$ element is fabricated on a copper-FR4 epoxy PCB and the performance is measured using a vector network analyzer (VNA) and a radiation pattern measurement setup. The simulation and measurement results are discussed in this section.

\subsection{Analysis of Resonant Frequency and Return Loss}

The frequency reponse of the return loss parameter (S11), obtained for the single patch antenna from simulation results, is shown in Fig. 4. It is observed that the antenna has a resoant frequency of $3.8 \mathrm{GHz}$ and a bandwidth of 140 $\mathrm{MHz}$. The return loss obtained at $3.8 \mathrm{GHz}$ is $-15 \mathrm{~dB}$.

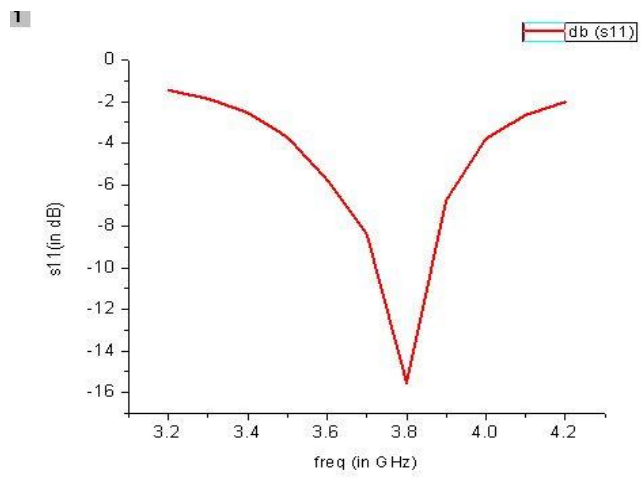

Fig.4. Return loss plot for E-shaped single patch antenna

In case of $1 \times 2$ array, the $\mathrm{S} 11$ vs. frequency plot is shown in Fig. 5. The resonating at desired frequency of this structure is also obtained at $3.8 \mathrm{GHz}$ with a bandwidth of $145 \mathrm{MHz}$. The return loss obtained at the resonating frequency is -25 dB.

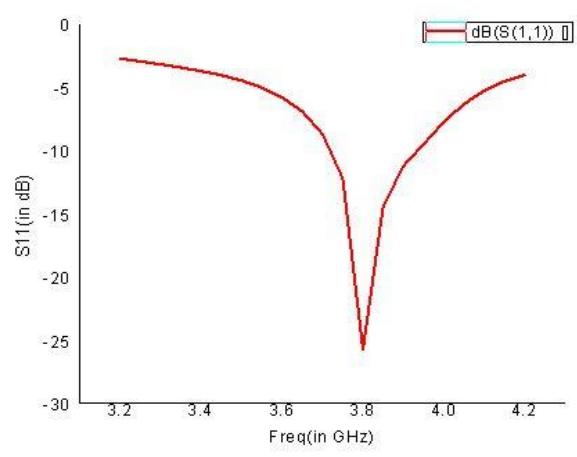

Fig.5. Comparison plot of $\mathrm{s} 11$ for single patch and $1 \times 2$ antenna 
Figure 6 shows the return loss for our desired $2 \times 2$ grid antenna. From the plot it can be seen that the antenna is delivering the minimum return loss of $-16.5 \mathrm{~dB}$, with a bandwidth of $160 \mathrm{MHz}$.

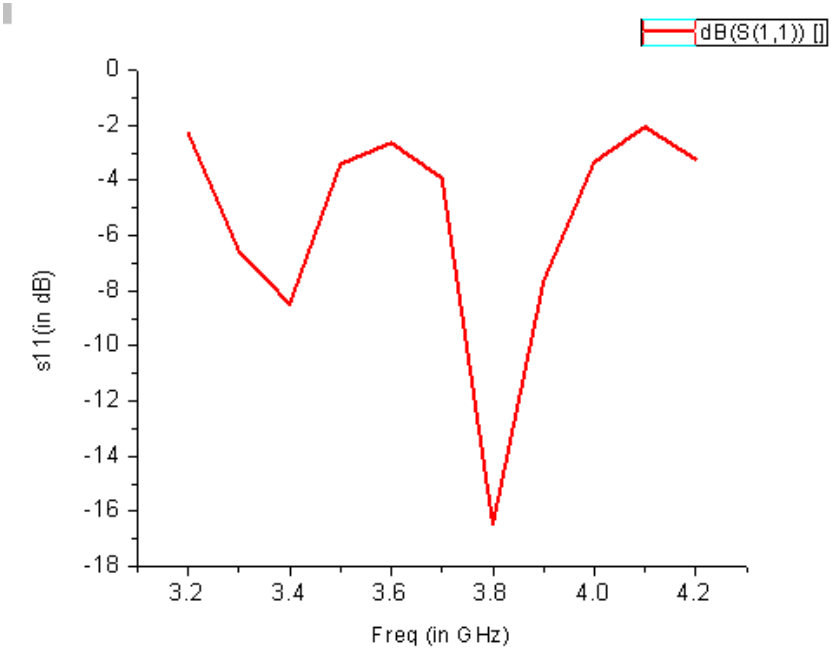

Fig.6 Return loss plot for E-shaped 2x 2 grid pattern antenna

\subsection{Antenna Gain and Radiation Pattern}

Obtaining enhanced gain and narrow beamwidth is the key advantage of constructing arrays from individual antenna elements. The radiation patterns of the far field gain of each of the three stages of the design are obtained from simulation results and compared.

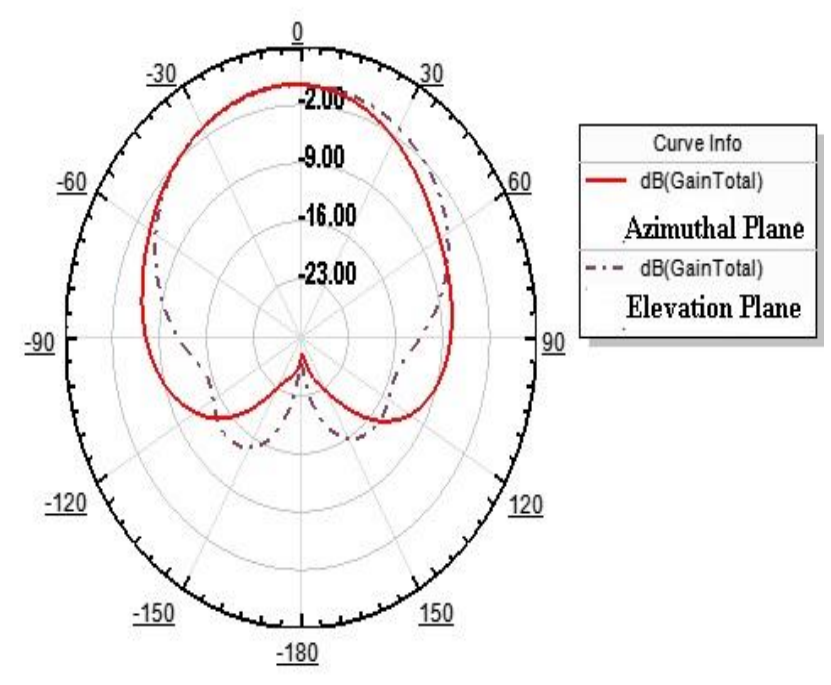

Fig.7. Radiation pattern of single patch antenna

Fig. 7, 8 and 9 show the far field radtion pattern in the azimuthal plane and elevation plane of the single patch antenna, the $1 \times 2$ array and the $2 \times 2$ grid structure respectively. It is observed that the single patch has a peak gain of $2.3 \mathrm{dBi}$ along the direction of the major lobe. The $1 \times 2$ array has a peak gain of $3.4 \mathrm{dBi}$ and the $2 \times 2$ grid has a peak gain of $6.5 \mathrm{dBi}$. Thus, the gain is enhanced as the number of elements in an array is more.

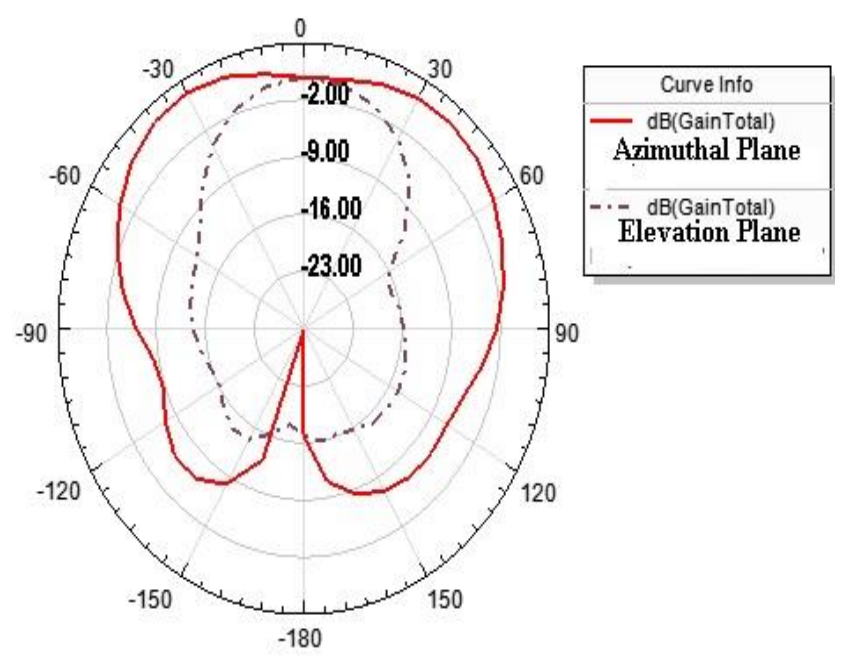

Fig.8. Radiation pattern of $1 \times 2$ patch antenna

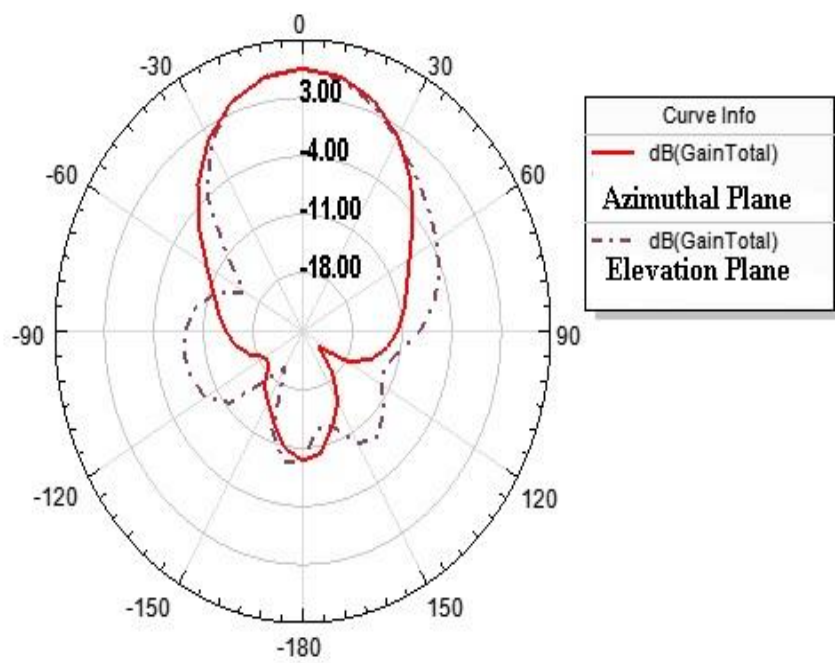

Fig.9. Radiation pattern of $2 \times 2$ Grid micro strip antenna

Comparing Fig. 7 and 8 , it is observed that the $1 \times 2$ array has a narrower beamwidth in the elevation plane compared to the single patch. However, in the azimuthal plane, the presence of a single broadside lobe is not observed. When Fig. 9 is compared with Fig. 7 it is observed that the major lobe of the $2 \times 2$ grid is significantly narrow as compared to the signle antenna element and the $1 \times 2$ array in both the azimuthal plane and the elevation plane.

\subsection{Fabrication and Measurement Results}

The final $2 \times 2$ grid antenna is fabricated on a copper-FR4 epoxy substrate as shown in Fig. 10. The frequency vs. S11 parameter plot obtained from VNA for the fabricated antenna is compared with the simulation result in Fig. 11.The measured far field radiation pattern of the antenna in the azimuthal plane and the elevation plane is shown in Fig. 12. 


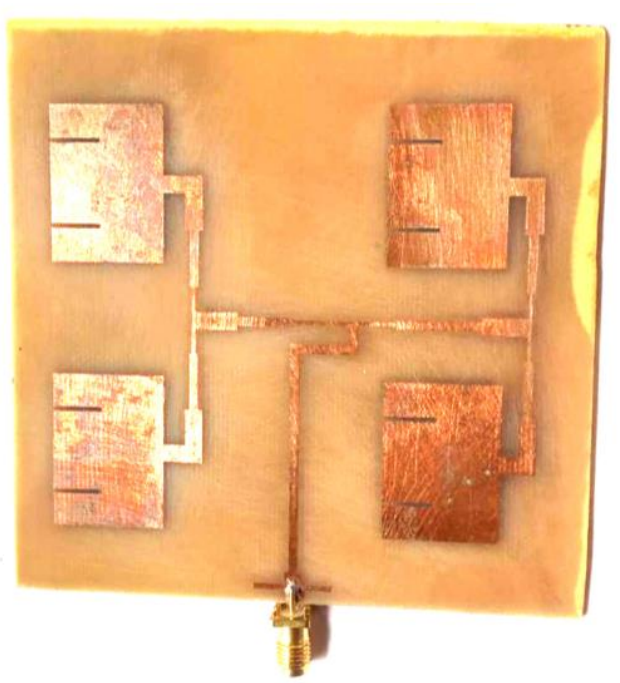

Fig. 10: The fabricated $2 \times 2$ grid array with E-shaped patch antenna

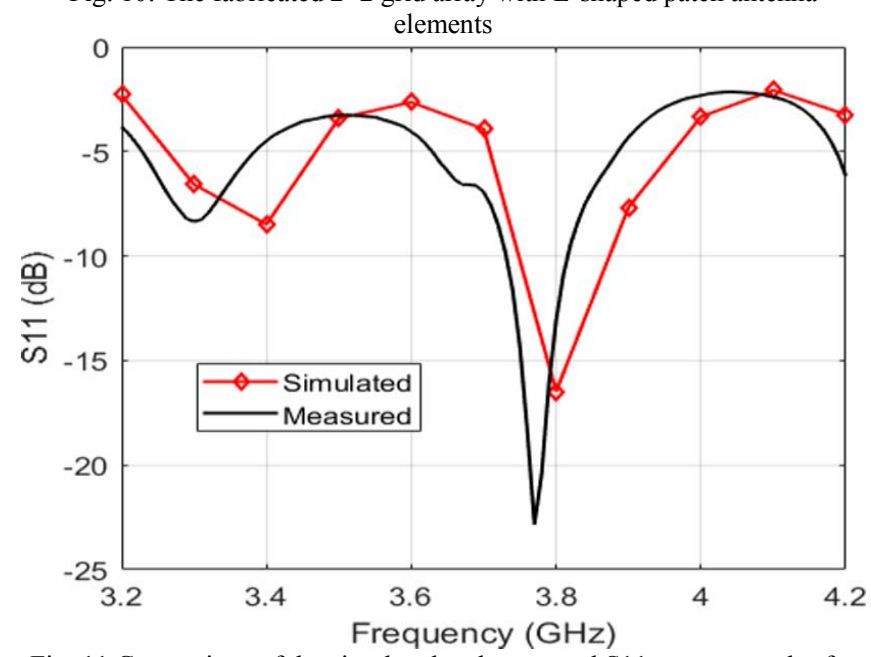

Fig. 11 Comparison of the simulated and measured S11 parameter plot for the $2 \times 2$ grid antenna

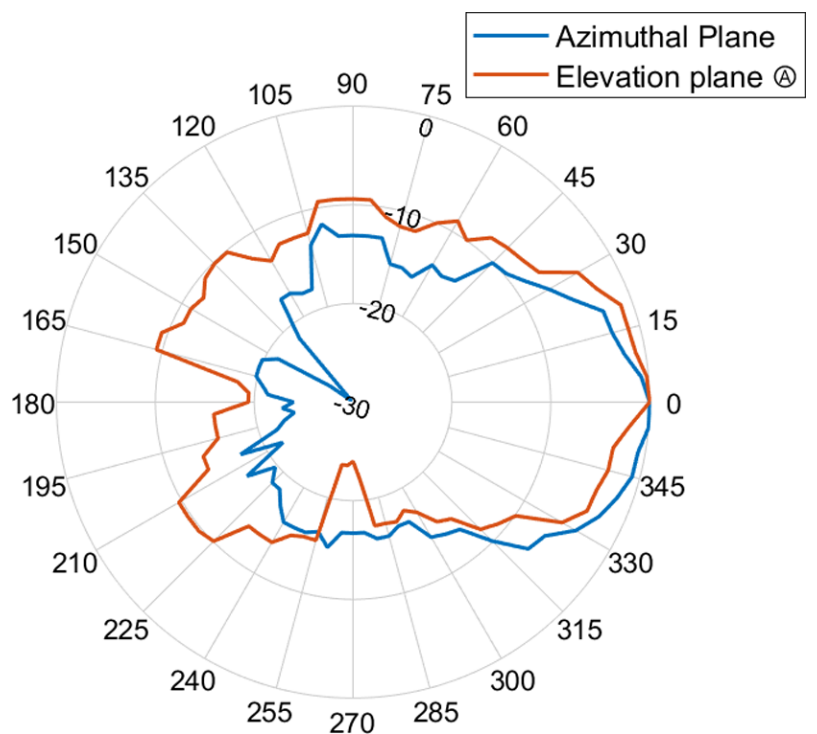

Fig. 12 Measured normalized radiation pattern of the $2 \times 2$ grid antenna in the azimuthal plane and the elevation plane

It is observed from the measurement results that the antenna resonates at $3.77 \mathrm{GHz}$ which is very close to $3.8 \mathrm{GHz}$. The radiation pattern of the fabricated grid antenna is almost similar to the simulation result.

\subsection{Discussion}

From the analysis of the resonant frequency and return loss presented in Section III (A), it is observed that the bandwidth of the antenna for the $1 \times 2$ array is more than that of the single patch antenna by around $5 \mathrm{MHz}$. The bandwidth further increases by about $15 \mathrm{MHz}$ when the $2 \times 2$ grid is constructed as compared to the $1 \times 2$ array. The $2 \times 2$ grid has a bandwidth of $160 \mathrm{MHz}$ which is significantly large in comparison with a single patch antenna.

In each case, a directional radiation pattern is obtained. The far field peak gain increases as the number of elements is more as discussed in Section III (B).

The comparison of these parameters are presented in Table II.

TABLE II. COMPARISON TABLE OF RESULTS

\begin{tabular}{lccc} 
Antenna & \multicolumn{3}{c}{ Antenna configuration type } \\
specifications & Single patch & $\mathbf{1 \times 2}$ array & $\mathbf{2 \times 2}$ grid \\
$\begin{array}{l}\text { Bandwidth } \\
\text { (in } \mathrm{MHz} \text { ) }\end{array}$ & 140 & 145 & 160 \\
Gain (in dBi ) & 2.3 & 3.4 & 6.5 \\
Radiation pattern & Directional & Directional & Directional
\end{tabular}

\section{Conclusions}

The proposed design and the experimental results show that by constructing a $2 \times 2$ grid of E-shaped patch antenna elements, the gain, the bandwidth and the directivity can be enhanced. The $2 \times 2$ grid yields a larger gain and bandwidth compared to a single patch antenna and a one-dimensional $1 \times 2$ array. From the comparative analysis of the radiation paterns as presented in Section II(B), it may be concluded that a one dimensional array of microstrip antennas enhance the directivity of the antenna only in the azimuthal plane. The proposed $2 \times 2$ grid of the antenna increases the directivity in both the azimuthal plane and the elevation plane. This is an additional advantage of a 2-dimensional grid of microstrip antennas.

\section{Acknowledgment}

The author expresses a heartiest gratitude to MHRD TEQIP III for their support .

\section{References}

[1] R. A. Abd-Alhameed1, D. Zhou1, c. H. See1, and P. S. Excel, "A Wire Grid Adaptive-Meshing Program forMicrostrip-Patch Antenna Designs sing aGenetic Algorithm", IEEE Antennas and Propagation Magazine Vol. 51, No.1, February 2009.

[2] K.Shrikar ,L.S Vinodh, B .Ramesh and K.P Vinay, "1x2 triangular shape Microstrip patch antenna array for WLAN applications with dgs structure," International journal of Innovative research in computer and Communication Engineering, vol. III ,issue III , March 2015, ISSN. 2320-9801

[3] S.P.Sinha ,M.Kumar, and J Gupta, "Design of $2 \times 2$ Shaped Rectengular Micro strip Array Antenna for GSM Application," International journal of Scientific and Engineering research, vol 6 ,issue 5, May 2015, ISSN. 2229-5518 
[4] J.George and B.Lethakumari, "Design and optimization of $2 \times 2$ corporate - series fed micro strip antenna array," International Journal of Engineering \& Technology, 7 (1.9), 2018, 84-87

[5] A. Slowik, M. Czyzewski and B. Slesicki, "The use of shunt-stubs in corporate-feeding network for S-band planar antenna array," 2018 22nd International Microwave and Radar Conference (MIKON), Poznan, 2018, pp.360-362. doi: 10.23919/MIKON.2018 .8405225

[6] H. M. M. Makkawi, "Performance evaluation of $1 \times 2$ patch antenna array based on power divider characterises," 2016 Conference of Basic Sciences and Engineering Studies (SGCAC), Khartoum, 2016, pp.6-12. doi: 10.1109/SGCAC.2016.7457998

[7] M. S. R Mohd Shah, M. Z. A Abdul Aziz and M. K. Suaidi, "Dual Linearly Polarized Microstrip Array Antenna", as a chapter in Trends in Telecommunications Technologies, Christos J Bouras (Ed.), pp. 367-388, 2010.

[8] B.T.P. Madhav, H. Khan, and V.G.N.S Prasad, "Microstrip 2x2 antenna on k15 liquid crystal substrate," International journal of applied engineering and research , vol. 6,number 9, pp. 1099$11041,2011$.

[9] Mei Sun, Yue Ping Zhang, Duixian Liu, Kai Meng Chua, and Lai Lai Wai, A Ball Grid Array Package With a Microstrip GridArray Antenna for a Single-Chip 60-GHz Receiver, IEEE Transactions on Antennas and Propagation, VOL. 59, NO. 6, JUNE 2011

[10] B.Akhilesh, G.Karthikeya, M.Sujatha, "Design of E-Shape Microstrip Patch Antenna for UltraWideband Applications", IOSR Journal of Electronics and Communication Engineering,vol 9,issue 2,pp 14-18, Apr 2014.

[11] D. M. Pozar, Microwave engineering. John Wiley \& Sons, 2009.

\section{Creative Commons Attribution License 4.0 (Attribution 4.0 International, CC BY 4.0)}

This article is published under the terms of the Creative Commons Attribution License 4.0

https://creativecommons.org/licenses/by/4.0/deed.en US 